\title{
Assessment of the Influence of Losses on the Performance of the Electromagnetic Time Reversal Fault Location Method
}

\author{
Reza Razzaghi, Member, IEEE, Gaspard Lugrin, Member, IEEE, Farhad Rachidi, Fellow IEEE, Mario \\ Paolone, Senior Member, IEEE
}

\begin{abstract}
Electromagnetic time reversal (EMTR) has been shown to be an efficient method for locating faults in AC and DC power grids. In the available literature, the back-propagation medium has been considered to have identical losses as the directtime medium. However, the telegrapher's equations describing the travelling wave propagation are time-reversal invariant if and only if inverted losses are considered in the back propagation phase. This paper presents an analysis of the impact of losses on the performance of the EMTR-based fault location method for power networks. In this respect, three back-propagation models are proposed, analyzed and compared. It is shown that a lossy back-propagation model, for which the wave equations are not rigorously time-reversal invariant, results in accurate fault locations. Finally, an EMTR fault location system based on the lossy back-propagation model and a fast electromagnetic transient simulation platform is developed and its performances validated.
\end{abstract}

Index Terms - Fault location, electromagnetic time reversal, electromagnetic transients, telegrapher's equations, transmission lines.

\section{INTRODUCTION}

The fault location problem has been extensively studied in the literature since 1950s [1] and numerous methods have been proposed. First, the subject was studied for transmission networks because of the importance of the fault location function in power systems operation and the difficulty of locating faults in meshed networks. Then, studies were extended to distribution networks as the power quality attracted an increased attention.

The proposed fault location methods, in general, fall into two general categories (e.g., [2]): (i) phasor-based (using voltage and current phasors), or (ii) travelling wave-based methods.

Using the voltage/current phasors at the line terminals is the most straightforward approach to estimate the fault location (e.g., [3], [4], [5], [6]). However, despite the straightforward

The authors are with the Electromagnetic Compatibility Laboratory (EMC) and Distributed Electrical Systems Laboratory (DESL) of the Swiss Federal Institute of Technology, Lausanne (EPFL), Switzerland, (e-mail: reza.razzaghi@epfl.ch, gaspard.lugrin@epfl.ch, farhad.rachidi@epfl.ch mario.paolone@epfl.ch). solutions provided by the phasor-based fault location methods, their accuracy might be affected by the fault resistance, configuration of the line, load unbalance, and the presence of distributed generation [2].

To overcome the limitations associated with the phasorbased fault location methods, travelling wave-based methods have been increasingly investigated in the literature (e.g., [7], [8], [9], [10]). These methods rely on the analysis of the highfrequency components of the fault-originated transient signals which are rather uninfluenced by the fault impedance [11].

Despite the more precise fault location accuracy of the travelling wave-based methods compared to phasor-based methods, their accuracy might still be affected by the following factors [2]:

- need of multiple observation points to avoid multiple solutions for the location of the fault.

- $\quad$ requirement of a precise time stamping for methods requiring multiple synchronized metering stations.

- loss of GPS signal impacting the fault location accuracy.

- requirement for relatively sophisticated signal processing analysis

To overcome the above-mentioned limitations associated with existing travelling wave-based fault location methods, an efficient fault location method based on the electromagnetic time reversal (EMTR) technique (e.g., [12], [13], [14]) has been proposed in [15]. It has been shown that telegrapher's equations describing the travelling wave propagation along the transmission lines are time-reversal invariant. Therefore, EMTR process, which is an effective procedure in focusing electromagnetic waves, has been used to locate faults in various power network topologies including inhomogeneous networks.

The EMTR method uses a straightforward procedure comprising three steps: (i) fault-originated transient signals are measured in a single observation point and then, time-reversed; (ii) a number of guessed fault locations (GFLs) is defined and for each GFL, the time-reversed signals are back-injected to the network back-propagation model and the Fault Current Signal Energy (FCSE) is calculated; (iii) according to the time reversal 
theory, the true fault location is characterized by a GFL which has the maximum FCSE. A review of the fault location method based on the EMTR is presented in section III.

Compared to other travelling wave-based fault location methods, the application of the EMTR method is straightforward for the case of inhomogeneous media such as mixed overhead and coaxial power cable lines. Moreover, it has been shown that EMTR method requires only a single observation point located at the secondary winding of a substation transformer to correctly identify the fault location [15]. Finally, the performances of this method is not influenced by the fault type and impedance [16]. The EMTR method has been successfully applied to different types of networks including inhomogeneous and radial distribution networks [15], series-compensated transmission lines [16], and more recently, multi-terminal HVDC networks [17].

As discussed in [15] and [18], the wave equations in transmission lines are time-reversal invariant for lossless lines. For the case of a lossy medium, the time-reversal invariance does not rigorously hold unless an inverted-loss medium is considered in the back-propagation model [18]. However, the simulation of the inverted-loss back-propagation model might be limited since commercial electromagnetic transient programs (such as EMTP) do not allow such implementations [19], [20].

In this respect, in this paper the effect of the losses associated with the line conductors and the ground on the accuracy of the EMTR-based fault location method is assessed. This paper is an extension of the preliminary study presented in [21] in which three different back-propagation models (lossless, lossy, and inverted-loss) were discussed.

The structure of the paper is the following: Section II presents the summary of the transmission line parameters, with particular reference to the losses. Section III presents the review of the EMTR-based fault location method. Section IV presents the definition of the three back-propagation models as well as the assessment of the impact of these models on the accuracy of the EMTR fault location method for the case of a single-wire line above a ground plane of finite conductivity. Section IV presents an analysis considering more realistic case studies such as an inhomogeneous network and a radial power grid. Section $\mathrm{V}$ describes the practical implementation of the fault location method by presenting the developed EMTR-based fault location platform. Section VI concludes the paper with final remarks.

\section{TRANSmission Lines Parameters: Single WiRE ABOVE A GROUND PLANE}

The presented analysis will be based on the transmission line (TL) theory. We make reference to Fig. 1 that represents the cross-section of a single-wire line above a ground plane. The wire has a radius $a$, a conductivity $\sigma_{w}$ and a relative permittivity $\varepsilon_{r w}$ and is located at a height $h$ above a homogenous ground of conductivity $\sigma_{g}$ and relative permittivity $\varepsilon_{r g} . \quad \sigma_{\text {air }}$ is the conductivity of the air.

Fig. 2 shows the equivalent circuit of a differential length of the considered line.

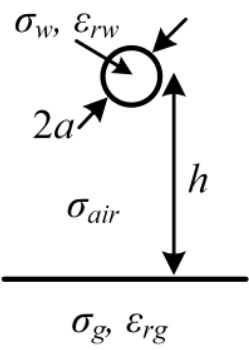

Fig. 1. Cross-section of a single-wire line above a ground plane.

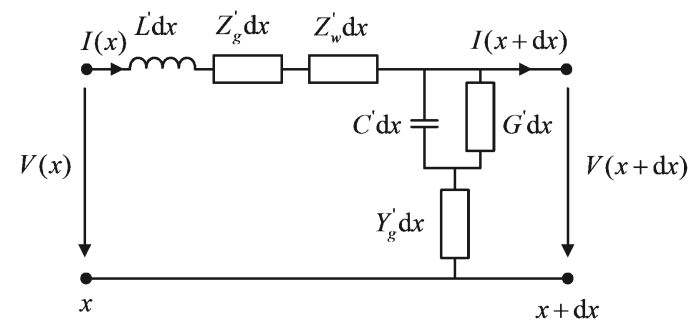

Fig. 2. Equivalent circuit of a single-wire line above a ground plane [14].

In Fig. 2, $L^{\prime}, C^{\prime}$ and $G^{\prime}$ are the per-unit-length (p.u.l) longitudinal inductance, transverse capacitance and transverse conductance, respectively, $Z_{w}^{\prime}$ is the p.u.l internal impedance of the wire, $\mathrm{Z}_{\mathrm{g}}^{\prime}$ and $\mathrm{Y}_{\mathrm{g}}^{\prime}$ are the per-unit-length ground impedance and admittance which account respectively for the losses associated with the penetration of magnetic and electric fields in the ground.

The expressions for the line parameters are well known and expressed as follows [22], [23].

$$
\begin{gathered}
L^{\prime}=\frac{\mu_{0}}{4 \pi} \cosh ^{-1}\left(\frac{\mathrm{h}}{a}\right) \quad C^{\prime}=\frac{2 \pi}{\varepsilon_{0} \cosh ^{-1}(\mathrm{~h} / \mathrm{a})} \\
Z_{w}{ }^{\prime}=\frac{\gamma_{w} \mathrm{I}_{0}\left(\gamma_{w} a\right)}{2 \pi a \sigma_{\mathrm{w}} \mathrm{I}_{1}\left(\gamma_{w} a\right)} \quad G^{\prime}=\frac{\sigma_{\text {air }}}{\varepsilon_{0}} C^{\prime} \quad Y_{g}{ }^{\prime} \cong \frac{\gamma_{g}{ }^{2}}{Z_{g}{ }^{\prime}} \\
Z_{g}{ }^{\prime}=\frac{\mathrm{j} \omega \mu_{0}}{\pi} \int_{0}^{\infty} \frac{e^{-2 h x}}{\sqrt{x^{2}+\gamma_{g}^{2}}+x} \mathrm{~d} x \cong \frac{\mathrm{j} \omega \mu_{0}}{2 \pi} \ln \frac{1+\gamma_{g} \mathrm{~h}}{\gamma_{g} \mathrm{~h}}
\end{gathered}
$$

where $I_{0}$ and $I_{1}$ are the modified Bessel functions of zero and first order, respectively. In (1), the propagation constants in the wire and in the ground are defined as follows.

$$
\begin{aligned}
& \gamma_{w}=\sqrt{\mathrm{j} \omega \mu_{0}\left(\sigma_{w}+\mathrm{j} \omega \varepsilon_{0} \varepsilon_{r w}\right)} \\
& \gamma_{g}=\sqrt{\mathrm{j} \omega \mu_{0}\left(\sigma_{g}+\mathrm{j} \omega \varepsilon_{0} \varepsilon_{r g}\right)}
\end{aligned}
$$

The total p.u.l longitudinal impedance and transverse admittance can be defined as

$$
\begin{aligned}
Z^{\prime} & =\mathrm{j} \omega L^{\prime}+Z_{w}{ }^{\prime}+Z_{g}{ }^{\prime} \\
Y^{\prime} & =\frac{\left(\mathrm{j} \omega C^{\prime}+G^{\prime}\right) Y_{g}{ }^{\prime}}{\mathrm{j} \omega C^{\prime}+G^{\prime}+Y_{g}{ }^{\prime}}
\end{aligned}
$$

from which the propagation constant can be calculated 


$$
\gamma=\alpha+\mathrm{j} \beta=\sqrt{Z^{\prime} Y^{\prime}}
$$

The expressions for the line parameters for a multi-conductor line can be found in [22].

It is worth mentioning that, the impact of the conductor and ground losses results in the attenuation of propagated transients and in the modification of the propagation speed, which are frequency dependent. Concerning the EMTR application, the latter is expected to be more critical since the timing is crucial.

\section{REVIEW OF THE EMTR-BASED FAULT LOCATION METHOD}

In this section, the EMTR-based fault location method proposed in [15] is briefly described. The method is composed of three steps:

I. The fault-originated voltage/current transient signals are recorded in the given observation point in the network:

$$
s_{i}(t), \quad t \in\left[t_{f}, t_{f}+T\right]
$$

where $s_{\mathrm{i}}(t)$ is the voltage/current fault-originated transient signal on conductor $i, t_{f}$ is the fault triggering time, and $T$ is the recording time window.

II. A set of a-priori Guessed Fault Locations (GFL) are defined:

$$
\text { GFL }: x_{f, m}, m=1, \ldots K
$$

The transient signals recorded in step I are timereversed and, for each GFL $\left(x_{f, m}\right)$, the network backpropagation model is simulated by back-injecting the time-reversed signals from the same observation point.

III. For each of the GFLs, the FCSE that corresponds to the energy of the currents flowing through the GFL is computed:

$$
\operatorname{FCSE}\left(x_{f, m}\right)=\sum_{p=1}^{M} \sum_{j=1}^{N}\left[i_{x_{f, m}}^{p}(j)\right]^{2}, \quad T=N \Delta t
$$

where $N$ is the number of samples, $\Delta t$ is the sampling time, and $p$ indicates the number of conductors in the line which are involved in the fault.

According to the time reversal theory, the FCSE is maximized at the real fault location. Thus, the maximum of the calculated FCSEs will indicate the real fault point:

$$
x_{f, \text { real }}=\left.\arg \right|_{x_{f, \mathrm{~m}}} \max \left\{\left(\operatorname{FCSE}\left(x_{f, m}\right)\right)\right\}
$$

\section{BACK-PROPAGATION MODELS}

\section{A. Lossless Back-Propagation Model}

In this model, losses are neglected in the time-reversed back propagation. Neglecting the losses corresponds to consider that the conductivity of the air is zero and the conductivities of the ground and of the wire tend to infinity

$$
\sigma_{\text {air }} \rightarrow 0 \quad \sigma_{w}, \sigma_{g} \rightarrow \infty
$$

As a result, the per-unit-length line parameters become

$$
\begin{array}{rlrl}
L_{1}{ }^{\prime} & =L^{\prime} & C_{1}{ }^{\prime} & =C^{\prime} \\
G_{1}{ }^{\prime} & =0 & Z_{w 1}{ }^{\prime}=0 \\
Z_{g 1}{ }^{\prime}=0 & Y_{g 1}{ }^{\prime} \rightarrow \infty
\end{array}
$$

The index ' 1 ' is used to characterize the parameters according to this first time-reversed back-propagation model. When introducing these parameters into (3) and (4), it results obviously into:

$$
\alpha_{1}=0
$$

And in general:

$$
\beta_{1} \neq \beta
$$

Since phase constant is not identical in the direct-time and back-propagation models, the propagation and phase velocity, in general, will be different. This might result in an imprecise fault location estimation.

Note that this model provides exact results only if the directtime propagation is also lossless.

\section{B. Lossy Back-Propagation Model}

In this model, losses are included in the back-propagation model and the conductivities are kept the same as during directtime propagation. Hence, assuming that the parameters of the line are perfectly known, the back-propagation line per-unitlength parameters are the same as during the direct propagation.

$$
\begin{aligned}
L_{2}{ }^{\prime} & =L^{\prime} & C_{2}{ }^{\prime} & =C^{\prime} \\
G_{2}{ }^{\prime} & =G^{\prime} & Z_{w 2}{ }^{\prime} & =Z_{w}{ }^{\prime} \\
Z_{g 2}{ }^{\prime} & =Z_{g}{ }^{\prime} & Y_{g 2}{ }^{\prime} & =Y_{g}{ }^{\prime}
\end{aligned}
$$

Even though a lossy medium is not time-reversal invariant, the lossy model also can result in an accurate fault location estimation. The reason is that the propagation speed, which is the crucial parameter in the time reversal process, is identical to the direct-time model. As a consequence, the propagation of the back-injected signals reflected from all the network boundaries will add up in phase at the real fault location. Therefore, it can be expected that this model provides more accurate fault location accuracy compared to the lossless model.

\section{Inverted-Loss Back-Propagation Model}

In this model, in order to maintain the equations timereversal invariant, the conductivities are inverted:

$$
\sigma_{\text {air }} \rightarrow-\sigma_{\text {air }} \quad \sigma_{w} \rightarrow-\sigma_{w} \quad \sigma_{g} \rightarrow-\sigma_{g}
$$

As a result, the propagation constants in the wire and in the ground become

$$
\gamma_{w 3}=-\gamma_{w}{ }^{*} \quad \gamma_{g 3}=-\gamma_{g}{ }^{*}
$$

when considering the appropriate choice for the root, namely keeping the same value for the imaginary part as in the propagation constants in direct time. The per-unit-length line parameters for time-reversed back propagation become 


$$
\begin{array}{cc}
L_{3}{ }^{\prime}=L^{\prime} & C_{3}{ }^{\prime}=C{ }^{\prime} \\
G_{3}{ }^{\prime}=-G^{\prime} & Z_{w 3}{ }^{{ }^{\prime}}=-Z_{w}{ }^{{ }^{*}} \\
Z_{g 3}{ }^{\prime}=-Z_{g 1}{ }^{{ }^{\prime}} & Y_{g 3}{ }^{{ }^{\prime}}=-Y_{g}{ }^{{ }^{*}}
\end{array}
$$

Parameters in (16) do not correspond to a physical model as the line itself becomes active and gives energy to the signal that is propagating along it. However, it can be numerically implemented.

This model is equivalent to invert the real part of $\gamma$ :

$$
\gamma_{3}=-\alpha+j \beta=-\gamma^{*}
$$

A complete computation of (15), (16) and (17) can be found in Appendix 1. For this model, it can readily be shown that the telegrapher's equations are time-reversal invariant. As a consequence, assuming the knowledge of the transmission line parameters, this model results in an exact fault location.

\section{Summary}

The mathematical expressions for the line parameters associated with the considered back-propagation models are summarized in Table I. As it can be seen in this table, the p.u.l. inductance and capacitance of the line are not modified by the back-propagation models. The models only affect the parameters corresponding to losses, namely the p.u.l. impedances and admittances.

TABLE I. LLINE PARAMETERS ACCORDING TO THE BACK-PROPAGATION MODELS.

\begin{tabular}{c|ccc} 
Parameter & $\begin{array}{c}\text { Lossless } \\
\text { Model }\end{array}$ & $\begin{array}{c}\text { Lossy } \\
\text { Model }\end{array}$ & $\begin{array}{c}\text { Inverted- } \\
\text { loss Model }\end{array}$ \\
\hline$L^{\prime}$ & $L^{\prime}$ & $L^{\prime}$ & $L^{\prime}$ \\
\hline$C^{\prime}$ & $C^{\prime}$ & $C^{\prime}$ & $C^{\prime}$ \\
\hline$G^{\prime}$ & 0 & $G^{\prime}$ & $-G^{\prime}$ \\
\hline$Z_{w}{ }^{\prime}$ & 0 & $Z_{w}{ }^{\prime}$ & $-Z_{w}{ }^{*}$ \\
\hline$Z_{g}{ }^{\prime}$ & 0 & $Z_{g}{ }^{\prime}$ & $-Z_{g}{ }^{*}$ \\
\hline$Y \mathrm{~g}^{\prime}$ & $\infty$ & $Y \mathrm{~g}^{\prime}$ & $-Y^{\prime}{ }^{\prime *}$
\end{tabular}

\section{E. Comparison of the Back-Propagation Models}

In order to assess the performances of the three backpropagation models, a simple power network composed of a 10 $\mathrm{km}$ single-wire overhead line above a finitely-conducting ground is considered.

By using the EMTR-based fault location method proposed in [6], the performances of the three back-propagation models are analysed in frequency-domain. The fault is considered at $8 \mathrm{~km}$ form the left terminal where the voltage transient generated by the fault is recorded and the conductor and ground losses are taken into account in the direct time fault simulations. The transmission line parameters are given in Table II. p.u.l parameters of the line were computed using expressions given in Section II. The ground conductivity is varied in the range of $0.001-1 \mathrm{~S} / \mathrm{m}$ and the for each value, the fault location error is computed.

Fig. 3 shows the estimated fault location error as a function of the ground conductivity for the 3 back-propagation models described earlier. It can be seen that the lossy and inverted-loss models provide zero error in the estimated fault location for different ground conductivities. Nevertheless, the lossless backpropagation model is not able to provide accurate fault location.

TABLE II. PARAMETERS OF THE LINE.

\begin{tabular}{ll}
\multicolumn{1}{c}{ Parameter } & \multicolumn{1}{c}{ Value } \\
\hline Height above the ground & $10 \mathrm{~m}$ \\
\hline Diameter of the wire & $1 \mathrm{~cm}$ \\
\hline Conductivity of the wire (copper) & $5.8 \cdot 10^{7} \mathrm{~S} / \mathrm{m}$ \\
\hline Relative permittivity of the ground & 10 \\
\hline Conductivity of the ground & $1-10^{-3} \mathrm{~S} / \mathrm{m}$ \\
\hline Terminal resistances & $50 \mathrm{k} \Omega$
\end{tabular}

The location error obviously depends on the line losses (essentially determined by the ground [24]). For a ground conductivity of $10^{-3} \mathrm{~S} / \mathrm{m}$, the location error is about $1.2 \mathrm{~km}$. The obtained results are consistent with those related to the use of EMTR to locate lightning discharges [25].

An important observation is that, even though in the lossy back-propagation model the wave equations are not strictly time-reversal invariant, the model results in an accurate fault location estimation. The importance of this result is that it makes possible to use commercial EMTP simulation programs to perform the EMTR fault location process.

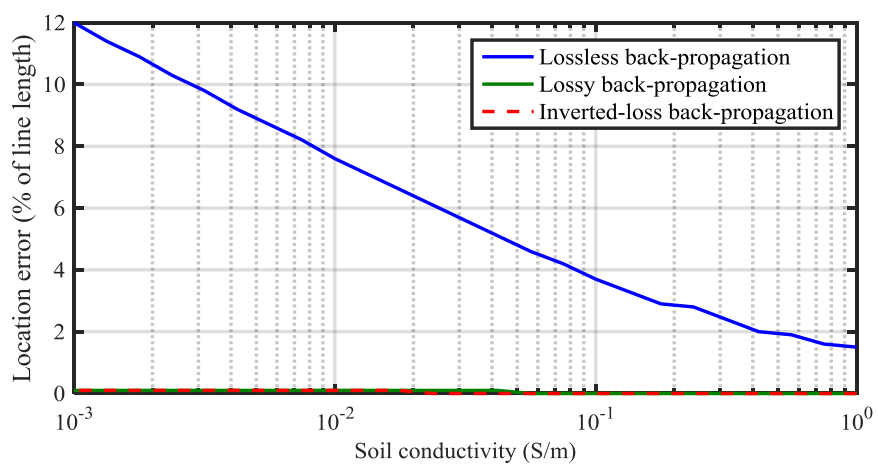

Fig. 3. Location error as a function of the soil conductivity, for the three proposed models.

\section{ApPlication to Realistic POWER NeTWORKS}

In this section, a further assessment of the performance of the back-propagation models considering realistic power network topologies is presented. Note that since the simulations are carried out using a commercial electromagnetic transients (EMT) simulation environments (EMTP-RV), it is not possible to consider the inverted-loss model. Therefore, for these case studies only the lossy and lossless models are considered.

\section{A. Inhomogeneous Network Composed of an Overhead Line and an Underground Cable}

The first considered case study is an inhomogeneous network. As shown in Fig. 4, the network is composed of an overhead line and an underground cable.

The overhead line and the cable lengths are $9 \mathrm{~km}$ and $3 \mathrm{~km}$, respectively and the network is simulated in EMTP-RV [19], [20] simulation environment. The overhead line and the cable parameters correspond to typical geometries of $230 \mathrm{kV}$ lines 
and cables and they are summarized in Appendix 2.

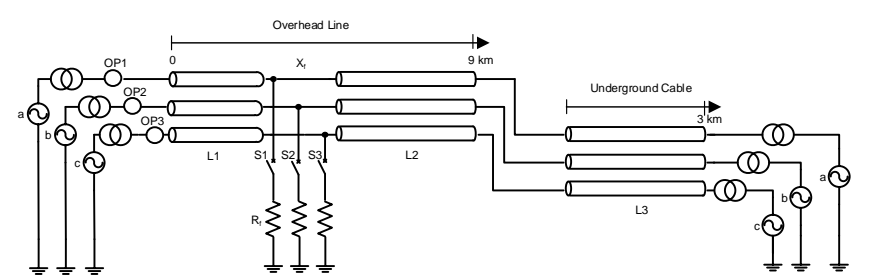

Fig. 4. The schematic representation of the inhomogeneous network simulated in EMTP-RV simulation environment.

The line and the cable ends are terminated to power transformers represented, as discussed in [15], by high impedances. All the fault transients are recorded at the three observation points (OP1, OP2, OP3) corresponding to the three conductors of the line (left terminal).

To study the impact of the back-propagation models on the accuracy of the EMTR fault location methods different fault types (i.e., three-phase, phase-to-phase, and phase-to-ground) are considered. The back-propagation simulations are performed using lossy and lossless models described in Section III. In the lossless model for the back propagation, two subcases are considered: (i) lossless conductors (but taking into account ground losses), and (ii) lossless ground (but taking into account the losses in the conductors.

Fig. 5 and Fig. 6 show the FCSE as a function of GFL for a three-phase-to-ground and phase-to-phase faults, respectively. The real fault location is at $7 \mathrm{~km}$ (overhead line) and the ground conductivity is $\sigma_{\mathrm{g}}=10^{-2} \mathrm{~S} / \mathrm{m}$.

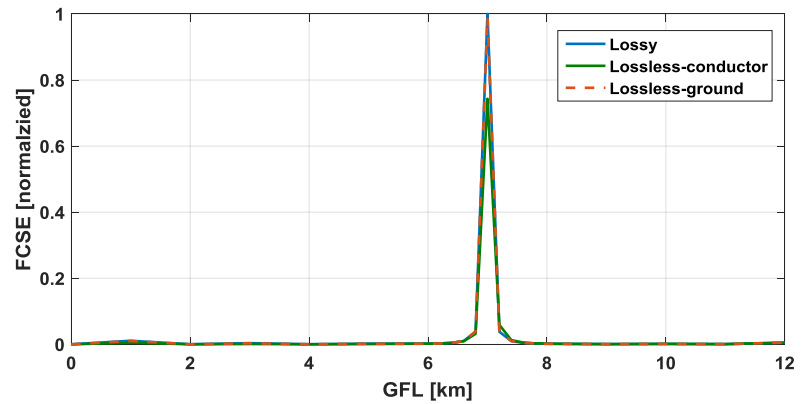

Fig. 5. FCSE as a function of GFL for three back-propagation models for different back-propagation models: (i) lossy, (ii) lossless conductors, and (iii) lossless ground. Three-phase fault at $7 \mathrm{~km}$ and $\sigma_{\mathrm{g}}=10^{-2} \mathrm{~S} / \mathrm{m}$.

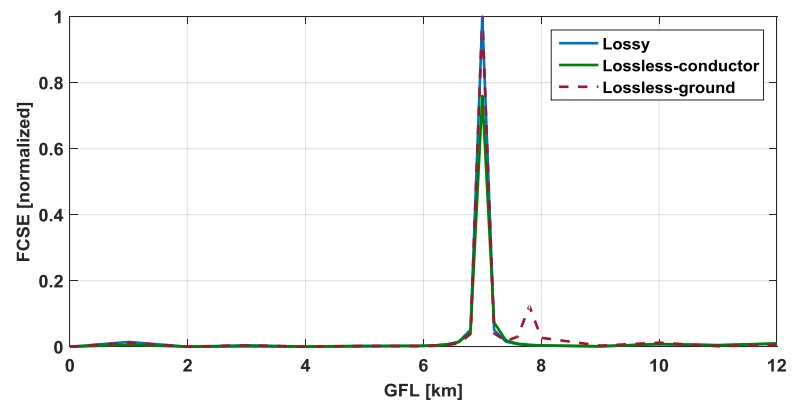

Fig. 6. FCSE as a function of GFL for three back-propagation models for different back-propagation models: (i) lossy, (ii) lossless wires, and (iii) lossless ground. Phase-to-phase fault at $7 \mathrm{~km}$ and $\sigma_{\mathrm{g}}=10^{-2} \mathrm{~S} / \mathrm{m}$.

It can be observed that for these fault types neglecting the ground and conductors' losses does not have significant influence on the accuracy of the fault location method.
The reason is that for these fault types, the ground mode (zero mode) is not involved in the fault current path. Therefore, the ground losses do not impact the fault current and as a consequent, the FCSE. Furthermore, for power systems the conductor losses are negligible compared to the ground losses.

For the case of phase-to-ground faults, the ground mode is involved in the fault current path. As known, ground mode is characterized by longer travel time, higher surge impedance and higher line resistance than the metallic modes. Therefore, neglecting the ground losses can have larger influence on the EMTR fault location accuracy. Fig. 7 shows the FCSE as a function of different GFL along the line for a phase-to ground fault at $7 \mathrm{~km}$. Similar to the previous case, the simulation is done considering the three different back-propagation models. It can be observed that neglecting ground losses impacts the accuracy of the fault location (the estimated fault location is 7.4 $\mathrm{km})$.

To further study the impact of the ground conductivity, the same simulation is performed again by considering a ground conductivity $\sigma_{\mathrm{g}}=10^{-3} \mathrm{~S} / \mathrm{m}$. Fig. 8 shows the FCSE as a function of GFL for a phase-to-ground and fault and for three different back-propagation models. By comparing Fig. 7 and Fig. 8 it can be seen that decreasing the ground resistivity deteriorates the fault location accuracy (the estimated fault location is $7.6 \mathrm{~km}$ ).

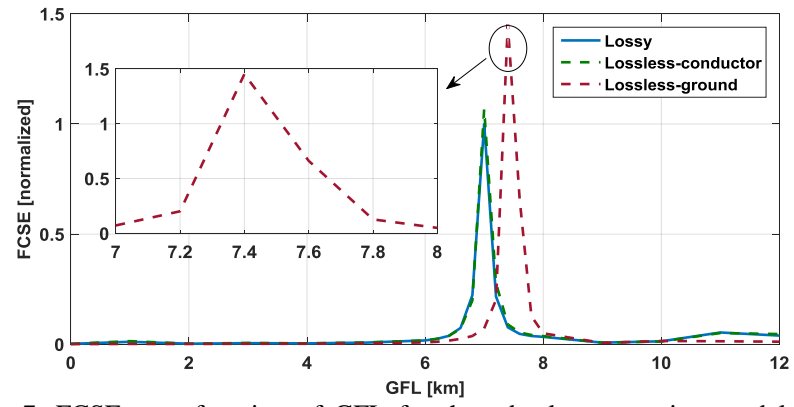

Fig. 7. FCSE as a function of GFL for three back-propagation models for different back-propagation models: (i) lossy, (ii) lossless conductors, and (iii) lossless ground. Phase-to-ground fault at $7 \mathrm{~km}$ and $\sigma_{\mathrm{g}}=10^{-2} \mathrm{~S} / \mathrm{m}$.

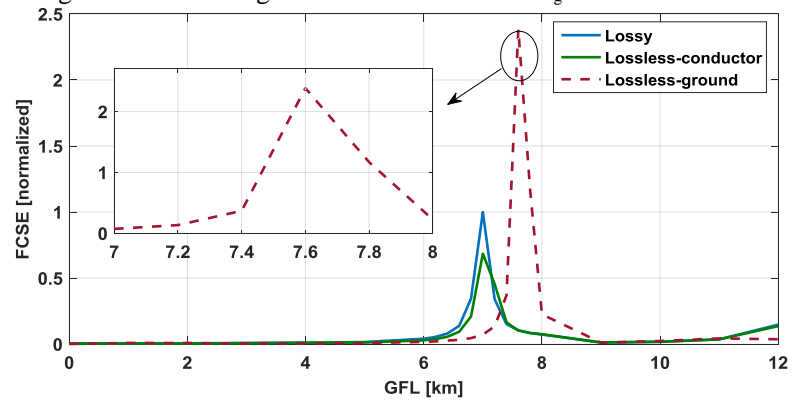

Fig. 8. FCSE as a function of GFL for three back-propagation models for different back-propagation models: (i) lossy, (ii) lossless conductors, and (iii) lossless ground. Phase-to-ground fault at $7 \mathrm{~km}$ and $\sigma_{\mathrm{g}}=10^{-3} \mathrm{~S} / \mathrm{m}$.

\section{B. Radial power networks}

The second case study is a radial network composed of 5 transmission lines. The network schematic is shown in Fig. 9. The EMTR fault location process is applied to this network by considering the three back-propagation models. Note that the EMTR process is performed using only a single observation point located at the secondary winding of Substation 1 (Fig. 9).

Similar to the previous case, the impact of the different back- 
propagation models is studied by considering different fault types and different ground conductivities. Fig. 10 shows the normalized FCSE as a function of GFL for a three-phase-toground and for (a) lossy, (b) lossless conductors, and (c) lossless ground, respectively.

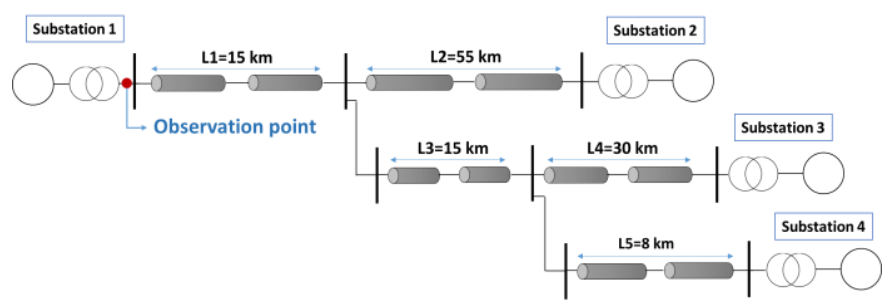

Fig. 9. Schematic representation of the radial network.

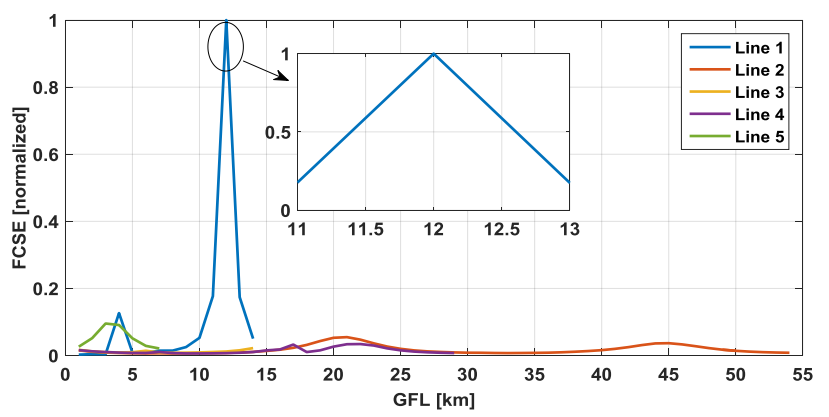

(a)

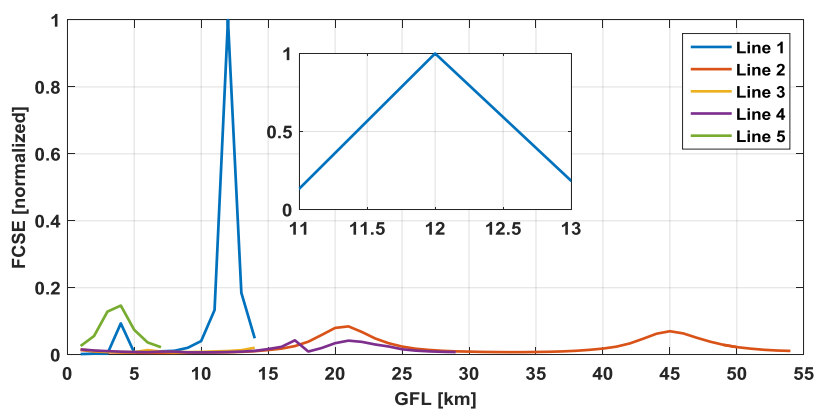

(b)

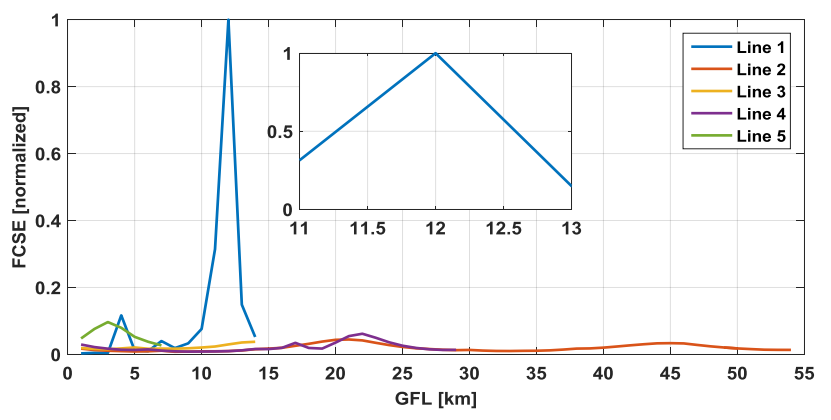

(c)

Fig. 10. FCSE as a function of GFL for three back-propagation models for different back-propagation models: (a) lossy, (b) lossless conductors, and (c) lossless ground. Three-phase fault at $12 \mathrm{~km}$ on line 1 and $\sigma_{\mathrm{g}}=10^{-3} \mathrm{~S} / \mathrm{m}$.

The real fault location is at $12 \mathrm{~km}$ on Line L1. The ground conductivity is $\sigma_{\mathrm{g}}=10^{-2} \mathrm{~S} / \mathrm{m}$. It can be observed that for a threephase fault, all the considered back-propagation models exhibit accurate fault location result.

A similar study is performed for the case of a phase-to-phase fault at $12 \mathrm{~km}$ along the first line. Fig. 11 shows the FCSE as a function of GFL for the considered back-propagation models.

Similar to the case of the three-phase fault, neglecting the losses does not impact the performance of the EMTR fault location method.

By following the same procedure, the analysis is also performed for the case of phase-to-ground fault. Fig. 12 shows the FCSE for the three back-propagation models. Unlike the three-phase and phase-to-phase faults, it can be seen that the ground losses have a non-negligible impact on the fault location accuracy for a phase-to-ground fault $(12.4 \mathrm{~km}$ instead of 12 $\mathrm{km})$. On the other hand, the lossy back-propagation model provides an accurate estimate of the fault location.

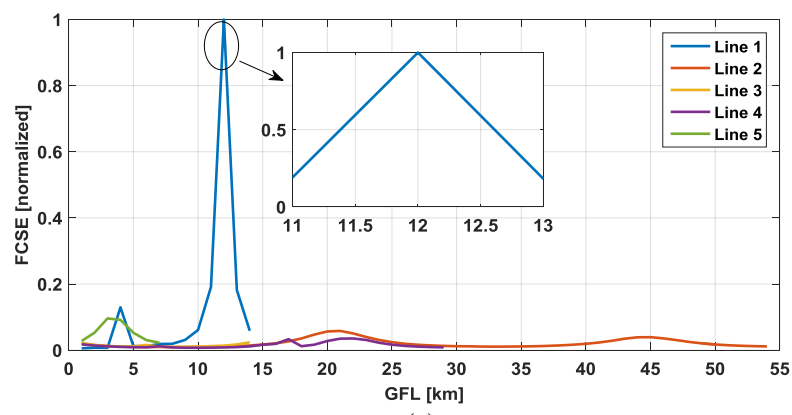

(a)

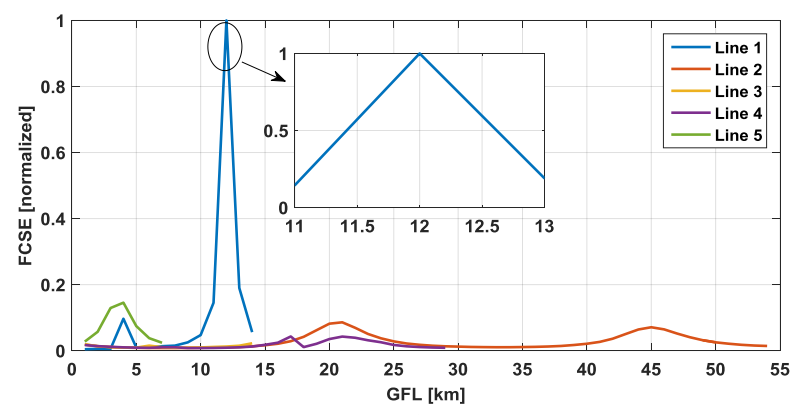

(b)

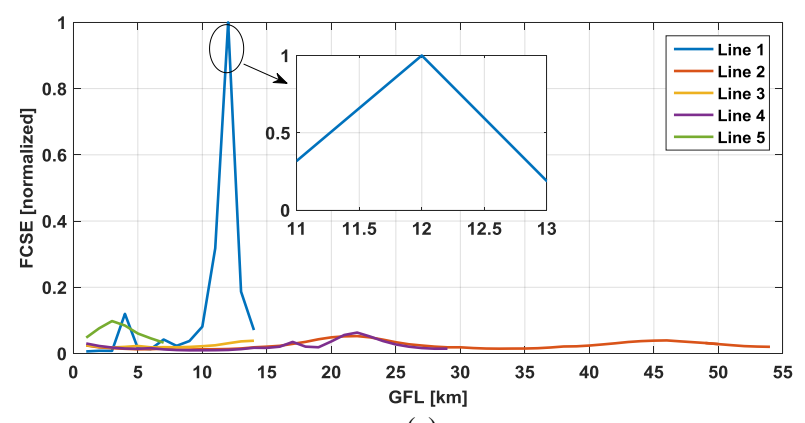

(c)

Fig. 11. FCSE as a function of GFL for three back-propagation models for different back-propagation models: (a) lossy, (b) lossless conductors, and (c) lossless ground. Phase-to-phase fault at $12 \mathrm{~km}$ on line 1 and $\sigma_{\mathrm{g}}=10^{-3} \mathrm{~S} / \mathrm{m}$. 


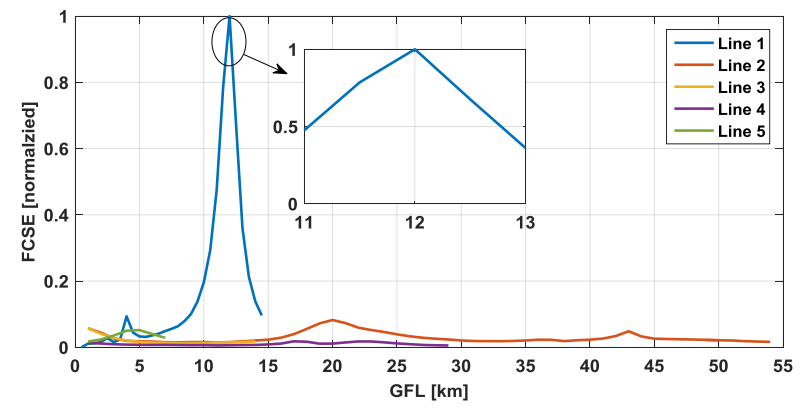

(a)

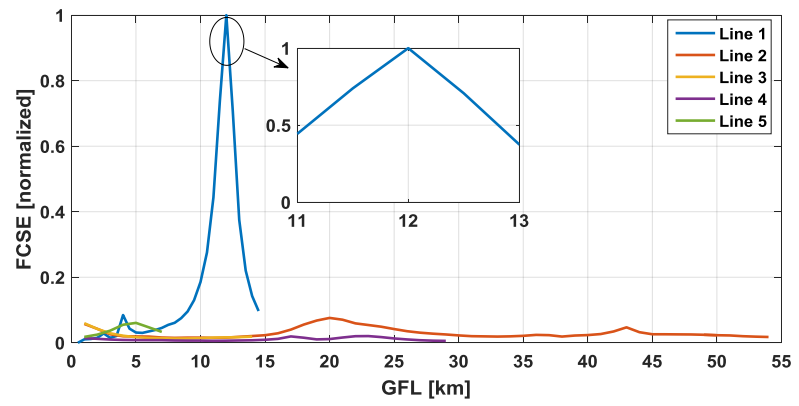

(b)

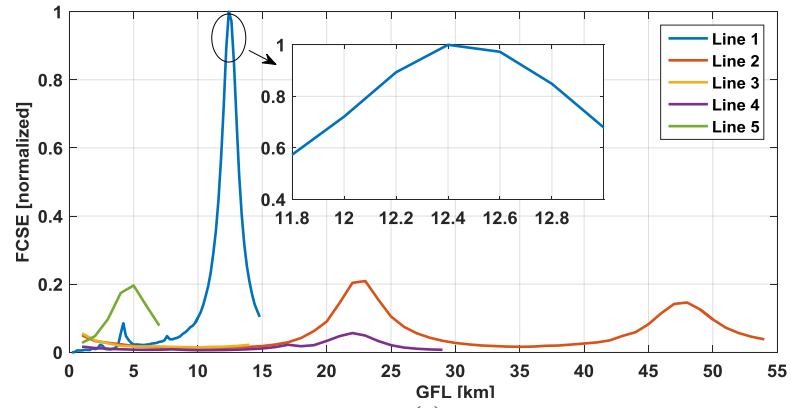

(c)

Fig. 12. FCSE as a function of GFL for three back-propagation models for different back-propagation models: (a) lossy, (b) lossless conductors, and (c) lossless ground. Phase-to-ground fault at $12 \mathrm{~km}$ on line 1 and $\sigma_{\mathrm{g}}=10^{-3} \mathrm{~S} / \mathrm{m}$.

\section{PRACTICAL IMPLEMENTATION OF THE EMTR-BASED FAULT LOCATION METHOD}

In order to evaluate the application of the EMTR-based fault location method in real world, a fault location platform based on this method is developed. The developed platform is based on an industrial hardware platform which integrates suitable processing capabilities as well as I/O modules for sampling the fault-originated transient signals. The use of the industrial hardware platform allows its straightforward coupling with the voltage/current sensors (with adequate sampling rate) and its deployment in the substation.

For a given network, the network topology as well as its parameters are the required information for the developed fault location platform.

Based on these information, the network model is realized in the specific real-time simulator which is dedicated to simulate the EMT in the network back-propagation model.

In what follows, the structure of the developed platform and its performance assessment are presented.

\section{A. The structure of the developed fault location platform}

The EMTR-based fault location method is based on multiple simulations of the back-propagation of time-reversed transients for different GFLs. Therefore, a fast EMT simulation platform is required to accelerate the process and enable its online application. To this end, an FPGA-based real-time EMT simulation platform is developed [26], [27]. Note that the analysis presented in the previous section demonstrated the adequacy of the lossy back-propagation model to identify the fault location with accuracy.

The developed FPGA-based real-time simulator (RTS) enables the possibility of accurately reproducing, in real-time, the electromagnetic waves propagating in transmission lines. The fast EMT simulation capability of the developed FPGARTS is exploited to perform the fault location process within short time periods.

The adopted hardware setup is composed of the National Instruments ComapctRIO platform, an industrial reconfigurable real-time embedded platform combining a micro-processor, an FPGA, and reconfigurable I/O modules. The micro-processor generates the data required by the FPGA real-time solver and the definition of the GFLs and simulation parameters (see [27] for more details). Then, these data are transferred to the FPGA which is dedicated to simulate the back-injection of the time-reversed signals and to calculate the FCSE corresponding to each GFL.

The developed fault location platform is able to (i) receive transient signals recorded at a given observation point of a power network via I/O modules (the sampling frequency of the available analog input modules are in the range of few hundreds of $\mathrm{kS} / \mathrm{s}$ to $1 \mathrm{MS} / \mathrm{s}$ ), (ii) apply the time reversal transformation to the recorded signals, and (iii) identify the fault location by applying the EMTR-based fault location procedure through multiple simulations corresponding to multiple GFLs in the network.

\section{B. Performance assessment}

In order to assess the performance of the developed fault location platform, the same network shown in Fig. 9 is considered. The network is composed of 5 transmission lines where each line is divided into two segments to provide the possibility of moving the fault location along its length. The fault location platform is considered to be installed at the substation where the fault-originated travelling waves are recorded. Thus, the fault location process is performed by using a single measurement station. The network model was implemented in the FPGA-RTS and a comprehensive performance evaluation was carried out by considering various fault location cases.

It is worth noting that, for this analysis real fault cases and the back-propagation of time-reversed signals are both simulated by the FPGA-RTS. More specifically, for each fault case, the network is simulated by the FPGA-RTS and the faultoriginated transient signals are recorded at the observation point. Then, using these signals and for each GFL, the back propagation of the time-reversed signals is simulated and the corresponding FCSE is calculated. The considered sampling 
frequency is $1 \mathrm{MHz}$.

Three category of fault types were considered (i.e., threephase, phase-to-phase, and phase-to-ground) and, for a given fault, the GFLs are considered along all the lines without the need for the knowledge of the faulty line. For each line, the fault location was moved with a location step of $1 \mathrm{~km}$ and for each real fault location, seven different fault types are considered. Then, the EMTR process was applied and the estimated fault location is identified. Table III shows the performance assessment of the developed fault location platform for the considered network.

TABLE III. PERFoRMANCE ASSESSMENT OF THE DEVELOPED FAULT LOCATION PLATFORM.

\begin{tabular}{|c|c|c|c|}
\hline Faulty line & Fault type & Nr. of faults & Nr. of mislocations \\
\hline \multirow{3}{*}{1} & $3 p h$ & 14 & 0 \\
\cline { 2 - 4 } & ph-ph & 52 & 0 \\
\cline { 2 - 4 } & ph-g & 52 & 0 \\
\hline \multirow{3}{*}{2} & $3 p h$ & 54 & 2 \\
\cline { 2 - 4 } & ph-ph & 162 & 6 \\
\cline { 2 - 4 } & ph-g & 162 & 2 \\
\hline \multirow{3}{*}{3} & $3 p h$ & 14 & 0 \\
\cline { 2 - 4 } & ph-ph & 52 & 0 \\
\cline { 2 - 4 } & ph-g & 52 & 0 \\
\hline \multirow{3}{*}{4} & $3 p h$ & 29 & 0 \\
\cline { 2 - 4 } & ph-ph & 87 & 0 \\
\cline { 2 - 4 } & ph-g & 87 & 0 \\
\hline \multirow{3}{*}{5} & $3 p h$ & 7 & 0 \\
\cline { 2 - 4 } & ph-ph & 21 & 0 \\
\cline { 2 - 4 } & ph-g & 21 & 0 \\
\hline
\end{tabular}

Based on this analysis, the following comments can be made:

1. The fault location system is able not only to assess the correct fault location but, also, is capable to identify the faulty line. This feature is of particular importance since it is performed by using a single-end measurement at the substation.

2. Among 866 simulated real fault cases, including different fault types, only in 10 cases $(1.15 \%)$, the system failed to correctly estimate the fault location.

3. Concerning the computation time, the average time to simulate a fault for a given GFL is $410 \mathrm{~ms}$. This execution time includes the times needed for: (i) updating the network parameters associated with each GFL, (ii) transferring the network information as well as the timereversed transient signals to the FPGA solver, (iii) calling the FPGA simulator to start simulation of the backpropagation model, and (iv) the time required by the FPGA simulator to run the back-propagation simulation and generate the FCSE corresponding to the GFL.

Note that the performance of the developed fault location platform was also evaluated in presence of the measurement noise. The same simulations were performed again by adding $40 \mathrm{~dB}$ SNR noise to the measured signals. The obtained results were exactly the same as the ones shown Table III indicating the robustness of the EMTR-based fault location method against the measurement noise.

\section{CONCLUSION}

The application of EMTR for the fault location problem is valid for lossless transmission lines in which the wave equations are time-reversal invariant. For the case of lossy lines, time-reversal invariance does not rigorously hold unless an inverted-loss medium is considered in the back-propagation model. In this respect, the impact of losses on the performance of the EMTR-based fault location method was analyzed. To this end, three back-propagation models were considered: (i) inverted-loss, (ii) lossless, and (iii) lossy.

Considering first, the case of a single-wire line above a conducting ground, we have shown that, as expected, an inverted-loss model provides a perfect estimation of the fault location. Additionally, the lossy back-propagation model also results in an accurate fault location.

The performance of the back-propagation models was further assessed by considering realistic power networks, namely an inhomogeneous (mixed overhead line and underground cable) network, and a radial grid. Different fault types were considered in the analysis: three-phase, phase-to-phase and phase-toground.

It was shown that, in general, a lossy model for the back propagation allows an accurate estimation of the fault location. For the cases of three-phase and phase-to-phase faults, the losses along the line can be neglected and a lossless backpropagation model would also provide accurate estimates for the fault location. For the phase-to-ground faults, losses in the ground play a significant role and thus, the lossless backpropagation model fails in accurately estimating the fault location.

The presented analysis demonstrates that a lossy backpropagation model, in which the wave equations in transmission lines are not rigorously time-reversal invariant, is able to accurately estimatethe location of the fault. Therefore, this analysis makes it possible to use commercial simulation programs to carry out EMTR fault location process.

Finally, a fault location platform was developed which integrates (i) the EMTR fault location method using the lossy back-propagation model, and (ii) a specific FPGA-based realtime simulator capable of fast simulation of electromagnetic transients propagations in transmission lines. The performance of the developed fault location platform was validated by making reference to a radial power network topology for different fault location cases with different fault types. The developed platform was able to identify the precise fault location within very short time period for nearly $99 \%$ of the considered cases. Furthermore, the platform was shown to be robust against several uncertainties including fault location, fault type and the measurement noise.

\section{ACKNOWLEDGEMENTS}

This work has been partially supported by SCCER-FURIES (Swiss Competence Center for Energy Research - Future Swiss Electrical Infrastructure). 


\section{APPENDIX 1: LINE PARAMETERS FOR THE INVERTED- LOSS BACK-PROPAGATION MODEL}

In this appendix, the derivation of expressions (15), (16) and (17) is presented.

First, the conductivities are reversed in the propagation constants of the wire (2), according to (14):

$$
\begin{aligned}
\gamma_{w 3} & =\sqrt{\mathrm{j} \omega \mu_{0}\left(-\sigma_{w}+\mathrm{j} \omega \varepsilon_{0} \varepsilon_{r w}\right)}= \\
& =\sqrt{-\mathrm{j} \omega \mu_{0}\left(\sigma_{w}-\mathrm{j} \omega \varepsilon_{0} \varepsilon_{r w}\right)}= \\
& =\sqrt{\left(\mathrm{j} \omega \mu_{0}\left(\sigma_{w}+\mathrm{j} \omega \varepsilon_{0} \varepsilon_{r w}\right)\right)^{*}}= \\
& =\left(\sqrt{\mathrm{j} \omega \mu_{0}\left(\sigma_{w}+\mathrm{j} \omega \varepsilon_{0} \varepsilon_{r w}\right)}\right)^{*}=-\gamma_{w}{ }^{*}
\end{aligned}
$$

when considering the appropriate choice for the root, namely keeping the same value for the imaginary part as in the propagation constant in direct time. In (18), the basic properties of the complex conjugate were used, that is for two complex numbers $z_{1}$ and $z_{2},\left(z_{1} z_{2}\right)^{*}=z_{1}{ }^{*} z_{2}{ }^{*}, \quad\left(z_{1}+z_{2}\right)^{*}=z_{1}{ }^{*}+z_{2}{ }^{*}$, and $\pm \sqrt{z_{1}^{*}}= \pm\left(\sqrt{z_{1}}\right)^{*}$.

The same derivation can be done for the propagation constant in the ground (2), by replacing the index ' $w$ ' by ' $g$ ' in (18).

$$
\gamma_{g 3}=\sqrt{\mathrm{j} \omega \mu_{0}\left(-\sigma_{g}+\mathrm{j} \omega \varepsilon_{0} \varepsilon_{r g}\right)}=-\gamma_{g}^{*}
$$

In (1), the p.u.l inductance and capacitance are not affected by the change of conductivity:

$$
\begin{aligned}
& L_{3}{ }^{\prime}=\frac{\mu_{0}}{4 \pi} \cosh ^{-1}\left(\frac{\mathrm{h}}{a}\right)=L^{\prime} \\
& C_{3}{ }^{\prime}=\frac{2 \pi}{\varepsilon_{0} \cosh ^{-1}(\mathrm{~h} / \mathrm{a})}=C^{\prime}
\end{aligned}
$$

Introducing this result and the change of conductivity in the expression of the p.u.l. conductance of the air leads to a change of sign:

$$
G_{3}{ }^{\prime}=\frac{-\sigma_{\text {air }}}{\varepsilon_{0}} C_{3}{ }^{\prime}=-G^{\prime}
$$

The p.u.l impedance of the wire for an inverted loss back propagation becomes

$$
\begin{aligned}
Z_{w 3}{ }^{\prime} & =\frac{-\gamma_{w}{ }^{*} \mathrm{I}_{0}\left(-\gamma_{w}{ }^{*} a\right)}{2 \pi a\left(-\sigma_{\mathrm{w}}\right) \mathrm{I}_{1}\left(-\gamma_{w}{ }^{*} a\right)}= \\
& =-\left(\frac{\gamma_{w} \mathrm{I}_{0}\left(\gamma_{w} a\right)}{2 \pi a \sigma_{\mathrm{w}} \mathrm{I}_{1}\left(\gamma_{w} a\right)}\right)^{*}=-Z_{w}{ }^{{ }^{*}}
\end{aligned}
$$

in which the symmetry properties of modified Bessel functions were used.

The p.u.l. impedance and admittance of the ground for an inverted loss back propagation become

$$
\begin{aligned}
& Z_{g 3}{ }^{\prime}=\frac{\mathrm{j} \omega \mu_{0}}{\pi} \int_{0}^{\infty} \frac{e^{-2 h x}}{\sqrt{x^{2}+\left(-\gamma_{g}{ }^{*}\right)^{2}}+x} \mathrm{~d} x= \\
& =-\left(\frac{\mathrm{j} \omega \mu_{0}}{\pi} \int_{0}^{\infty} \frac{e^{-2 h x}}{\sqrt{x^{2}+\gamma_{g}{ }^{2}}+x} \mathrm{~d} x\right)^{*}=-Z_{g}{ }^{\prime^{*}} \\
& Y_{g 3}{ }^{\prime} \cong \frac{\gamma_{g 3}{ }^{2}}{Z_{g 3}{ }^{\prime}}=\frac{\left(-{\gamma_{g}}^{*}\right)^{2}}{-Z_{g}{ }^{*}}=-\left(\frac{\gamma_{g}{ }^{2}}{Z_{g}{ }^{\prime}}\right)^{*}=-Y_{g}{ }^{\prime^{*}}
\end{aligned}
$$

The expressions for the total p.u.l impedance and admittance of the line can be readily obtained

$$
\begin{aligned}
Z_{3}{ }^{\prime} & =\mathrm{j} \omega L_{3}{ }^{\prime}+Z_{w 3}{ }^{\prime}+Z_{g 3}{ }^{\prime}=\mathrm{j} \omega L^{\prime}-Z_{w}{ }^{*}{ }^{*}-Z_{g}{ }^{* *}= \\
& =-\left(\mathrm{j} \omega L^{\prime}+Z_{w}{ }^{\prime}+Z_{g}{ }^{\prime}\right)^{*}=-Z^{{ }^{*}} \\
Y_{3}{ }^{\prime}= & \frac{\left(\mathrm{j} \omega C_{3}{ }^{\prime}+G_{3}{ }^{\prime}\right) Y_{g 3}{ }^{\prime}}{\mathrm{j} \omega C_{3}{ }^{\prime}+G_{3}{ }^{\prime}+Y_{g 3}{ }^{\prime}}=\frac{\left(\mathrm{j} \omega C^{\prime}-G^{\prime}\right)\left(-Y_{g}{ }^{\prime}\right)^{*}}{\mathrm{j} \omega C^{\prime}-G^{\prime}-Y_{g}{ }^{\prime *}}= \\
= & \frac{-\left(\mathrm{j} \omega C^{\prime}+G^{\prime}\right)^{*}\left(-Y_{g}{ }^{\prime}\right)^{*}}{-\left(\mathrm{j} \omega C^{\prime}+G^{\prime}+Y_{g}{ }^{\prime}\right)^{*}}=-Y^{{ }^{*}}
\end{aligned}
$$

Finally, the propagation constant of the line is given by

$$
\gamma_{3}=\sqrt{\left(-Z^{*}\right)\left(-Y^{\prime^{*}}\right)}=\left(\sqrt{Z^{\prime} Y^{\prime}}\right)^{*}=-\gamma^{*}
$$

when considering the choice for the root that keeps the same value for the imaginary part as in the direct time.

\section{APPENDIX 2: PARAMETERS OF THE LINE AND CABLE IN THE NETWORK PRESENTED IN FIG. 4.}

The p.u.l. series impedance and shunt admittance matrices for the line and cable of the inhomogeneous system shown in Fig. 4 and evaluated at their respective switching frequency are given by (27)-(30).

$$
Z_{\text {Line }}=\left[\begin{array}{ccc}
1.10+j 15.32 & 1.00+j 5.80 & 1.00+j 4.64 \\
1.00+j 5.80 & 1.09+j 15.33 & 1.00+j 5.80 \\
1.00+j 4.64 & 1.00+j 5.80 & 1.10+j 15.32
\end{array}\right] \frac{\Omega}{\mathrm{km}}
$$

$$
Y_{\text {Line }}=\left[\begin{array}{ccc}
2 \cdot 10^{-4}+j 67.53 & -j 16.04 & -j 7.91 \\
-j 16.04 & 2 \cdot 10^{-4}+j 70.12 & -j 16.04 \\
-j 7.91 & -j 16.04 & 2 \cdot 10^{-4}+j 67.53
\end{array}\right] \times 10^{-6} \frac{\mathrm{S}}{\mathrm{km}}
$$

$Z_{\text {Cable }}=$

$\left[\begin{array}{llllll}0.07+j 0.70 & 0.05+j 0.45 & 0.05+j 0.41 & 0.05+j 0.62 & 0.05+j 0.45 & 0.05+j 0.41\end{array}\right]$ $\begin{array}{lllllll}0.05+j 0.45 & 0.07+j 0.70 & 0.05+j 0.45 & 0.05+j 0.45 & 0.05+j 0.62 & 0.05+j 0.45\end{array}$ $\begin{array}{lllllll}0.05+j 0.41 & 0.05+j 0.45 & 0.07+j 0.70 & 0.05+j 0.41 & 0.05+j 0.45 & 0.05+j .62 & \Omega \\ 0.05+j 0.62 & 0.05+j 0.45 & 0.05+j 0.41 & 0.05+j 0.62 & 0.05+j 0.45 & 0.05+j 0.41\end{array}$ $\begin{array}{lllllll}0.05+j 0.62 & 0.05+j 0.45 & 0.05+j 0.41 & 0.03+j 0.62 & 0.05+j 0.45 & 0.05+j 0.41 & \mathrm{~km}\end{array}$ $\begin{array}{lllllll}0.05+j 0.45 & 0.05+j 0.62 & 0.05+j 0.45 & 0.05+j 0.45 & 0.03+j 0.62 & 0.05+j 0.45\end{array}$ $\left[\begin{array}{lllllll}0.05+j 0.41 & 0.05+j 0.45 & 0.05+j 0.62 & 0.05+j 0.41 & 0.05+j 0.45 & 0.03+j 0.62\end{array}\right]$

(29) 


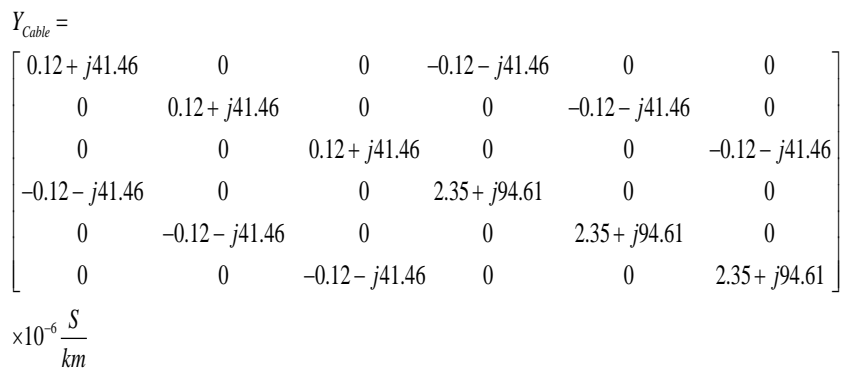

\section{REFERENCES}

[1] T. W. Stringfield, D. J. Marihart, and R. F. Stevens, "Fault Location Methods for Overhead Lines," Trans. Am. Inst. Electr. Eng. Part III Power Appar. Syst., vol. 76, no. 3, pp. 518-529, Apr. 1957.

[2] "IEEE Guide for Determining Fault Location on AC Transmission and Distribution Lines." 2005.

[3] T. Takagi, Y. Yamakoshi, M. Yamaura, R. Kondow, and T. Matsushima, "Development of a New Type Fault Locator Using the One-Terminal Voltage and Current Data," IEEE Trans. Power Appar. Syst., vol. PAS101, no. 8, pp. 2892-2898, Aug. 1982.

[4] M. Kezunovic and B. Perunicic, "Automated Transmission Line Fault Analysis Using Synchronized Sampling at Two Ends," in Proceedings of Power Industry Computer Applications Conference, 1995, pp. 407-413.

[5] G. Feng and A. Abur, "Fault Location Using Wide-Area Measurements and Sparse Estimation," IEEE Trans. Power Syst., vol. 31, no. 4, pp. 2938-2945, 2016.

[6] S. Gajare and A. Pradhan, "An Accurate Fault Location Method for MultiCircuit Series Compensated Transmission Lines," IEEE Trans. Power Syst., pp. 1-1, 2016.

[7] Y. G. Paithankar and M. T. Sant, "A New Algorithm for Relaying and Fault Location Based on Autocorrelation of Travelling Waves," Electr. Power Syst. Res., vol. 8, no. 2, pp. 179-185, Mar. 1985.

[8] P. McLaren and S. Rajendra, "Travelling-Wave Techniques Applied to the Protection of Teed Circuits:- Multi-Phase/Multi-circuit Sytstem," IEEE Trans. Power Appar. Syst., vol. PAS-104, no. 12, pp. 3551-3557, Dec. 1985.

[9] F. V. Lopes, K. M. Silva, F. B. Costa, W. L. A. Neves, and D. Fernandes, "Real-Time Traveling-Wave-Based Fault Location Using Two-Terminal Unsynchronized Data," IEEE Trans. Power Deliv., vol. 30, no. 3, pp. 1067-1076, Jun. 2015.

[10] R. J. Jalilzadeh Hamidi and H. Livani, "Traveling Wave-Based Fault Location Algorithm for Hybrid Multi-Terminal Circuits," IEEE Trans. Power Deliv., pp. 1-1, 2016.

[11] A. Borghetti, M. Bosetti, M. Di Silvestro, C. A. Nucci, and M. Paolone, "Continuous-wavelet transform for Fault Location in Distribution Power Networks: Definition of Mother Wavelets Inferred From Fault Originated Transients," IEEE Trans. Power Syst., vol. 23, no. 2, pp. 380-388, 2008.

[12] M. Fink, "Time Reversal of Ultrasonic Fields. I. Basic Principles.," IEEE Trans. Ultrason. Ferroelectr. Freq. Control, vol. 39, no. 5, pp. 555-66, Jan. 1992.

[13] J. de Rosny, G. Lerosey, M. Fink, J. De Rosny, G. Lerosey, and M. Fink, "Theory of Electromagnetic Time-Reversal Mirrors," IEEE Trans. Antennas Propag., vol. 58, no. 10, pp. 3139-3149, Oct. 2010.

[14] G. Lerosey, J. de Rosny, A. Tourin, A. Derode, G. Montaldo, and M. Fink, "Time Reversal of Electromagnetic Waves," Phys. Rev. Lett., vol. 92, no. 19, p. 193904, May 2004.

[15] R. Razzaghi, G. Lugrin, H. M. Manesh, C. Romero, M. Paolone, and F. Rachidi, "An Efficient Method Based on the Electromagnetic Time Reversal to Locate Faults in Power Networks," IEEE Trans. Power Deliv., vol. 28, no. 3, pp. 1663-1673, Jul. 2013.

[16] R. Razzaghi, G. Lugrin, M. Paolone, and F. Rachidi, "On the Use of Electromagnetic Time Reversal to Locate Faults in Series-Compensated Transmission Lines," in 2013 IEEE Grenoble Conference, 2013, pp. 1-5.

[17] R. Razzaghi, M. Paolone, F. Rachidi, J. Descloux, B. Raison, and N. Retiere, "Fault location in Multi-Terminal HVDC Networks Based on Electromagnetic Time Reversal with Limited Time Reversal Window," in 2014 Power Systems Computation Conference, 2014, pp. 1-7.
[18] F. Rachidi and M. Rubinsein, "Time Reversal of Electromagnetic Fields and its Application to Lightning Location," in 2013 International Symposium on Lightning Protection (XII SIPDA), 2013, pp. 378-383.

[19] H. Dommel, "Digital Computer Solution of Electromagnetic Transients in Single-and Multiphase Networks," IEEE Trans. Power Appar. Syst., vol. PAS-88, no. 4, pp. 388-399, Apr. 1969.

[20] J. Mahseredjian, S. Dennetière, L. Dubé, B. Khodabakhchian, and L. Gérin-Lajoie, "On a New Approach for the Simulation of Transients in Power Systems," Electr. Power Syst. Res., vol. 77, no. 11, pp. 1514-1520, Sep. 2007.

[21] L. Gaspard, R. Razzaghi, F. Rachidi, and M. Paolone, "Electromagnetic Time Reversal Applied to Fault Detection: the Issue of Losses," in Joint IEEE International Symposium on Electromagnetic Compatibility and EMC Europe, 2015.

[22] C. A. Nucci and F. Rachidi, "Interaction of Electromagnetic Fields Generated by Lightning with Overhead Electrical Networks," in The Lightning Flash, V. Cooray, Ed. London: IET, 2004, pp. 559-610.

[23] F. Rachidi, “A Review of Field-to-Transmission Line Coupling Models With Special Emphasis to Lightning-Induced Voltages on Overhead Lines," IEEE Trans. Electromagn. Compat., vol. 54, no. 4, pp. 898-911, Aug. 2012.

[24] F. Rachidi, C. A. Nucci, M. Ianoz, and C. Mazzetti, "Influence of a Lossy Ground on Lightning-Induced Voltages on Overhead Lines," IEEE Trans. Electromagn. Compat., vol. 38, no. 3, pp. 250-264, 1996.

[25] G. Lugrin, N. M. Parra, F. Rachidi, M. Rubinstein, and G. Diendorfer, "On the Location of Lightning Discharges Using Time Reversal of Electromagnetic Fields," IEEE Trans. Electromagn. Compat., vol. 56, no. 1, pp. 149-158, Feb. 2014.

[26] R. Razzaghi, M. Paolone, and F. Rachidi, "A general purpose FPGAbased real-time simulator for power systems applications," in 2013 4th IEEE/PES Innovative Smart Grid Technologies Europe, ISGT Europe 2013, 2013.

[27] R. Razzaghi, M. Mitjans, F. Rachidi, and M. Paolone, "An automated FPGA real-time simulator for power electronics and power systems electromagnetic transient applications," Electr. Power Syst. Res., vol. 141, pp. 147-156, 2016.

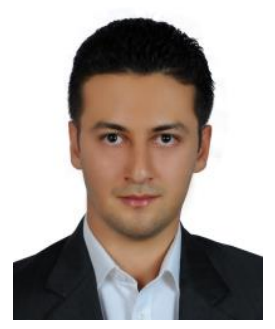

Reza Razzaghi (S'10) received the B.Sc. and M.Sc. degrees from the University of Tehran, Iran, in 2009 and 2011, respectively and the Ph.D. degree from the Swiss Federal Institute of Technology of Lausanne (EPFL), Switzerland in 2016, all in electrical engineering. Currently, he is a postdoctoral research associate at the Distributed Electrical System Laboratory (DESL) of the Swiss Federal Institute of Technology of Lausanne (EPFL), Switzerland. His research interests include real-time simulation of power systems, power system protections and power system transients.

Dr. Razzaghi was a recipient of the "Basil Papadias" Best Paper Award at the 2013 IEEE Powertech conference in Grenoble.

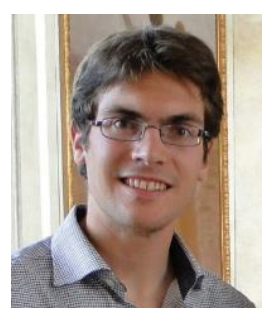

Gaspard Lugrin received the $\mathrm{PhD}$ degree in electrical engineering from the Swiss Federal Institute of Technology of Lausanne (EPFL), Switzerland in 2016. His research interests include different topics dealing with electromagnetic compatibility such as electromagnetic coupling to structures, location of transient sources using time reversal and high-power electromagnetics. 


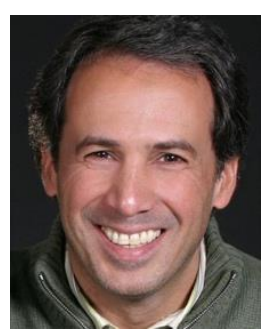

Farhad Rachidi (M'93-SM'02-F'10) received the M.S. degree in electrical engineering and the Ph.D. degree from the Swiss Federal Institute of Technology, Lausanne, in 1986 and 1991 respectively. He worked at the Power Systems Laboratory of the same institute until 1996. In 1997, he joined the Lightning Research Laboratory of the University of Toronto in Canada and from April 1998 until September 1999, he was with Montena EMC in Switzerland. He is currently a Titular Professor and the head of the EMC Laboratory at the Swiss Federal Institute of Technology (EPFL), Lausanne, Switzerland. Dr. Rachidi served as the Vice-Chair of the European COST Action on the Physics of Lightning Flash and its Effects (20052009), the Chairman of the 2008 European Electromagnetics International Symposium (EUROEM), the President of the International Conference on Lightning Protection (2008-2014), and the Editor-in-Chief of the IEEE TRANSACTIONS ON ELECTROMAGNETIC COMPATIBILITY (2013-2015). He is presently a member of the Advisory Board of the IEEE TRANSACTIONS ON Electromagnetic Compatibility and the President of the Swiss National Committee of the International Union of Radio Science (URSI).

Farhad Rachidi is the author or coauthor of 150 scientific papers published in peer-reviewed journals and over 350 papers presented at international conferences. He has received numerous awards including the 2005 IEEE EMC Technical Achievement Award, the 2005 CIGRE Technical Committee Award and the 2006 Blondel Medal from the French Association of Electrical Engineering, Electronics, Information Technology and Communication (SEE), and the 2016 Karl Berger Award. In 2014, Farhad Rachidi was conferred the title of Honorary Professor of the Xi'an Jiaotong University in China.

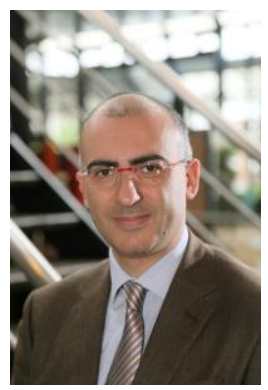

Mario Paolone (M'07-SM'10) received the M.Sc. (with honors) and the Ph.D. in electrical engineering from the University of Bologna, Italy in 1998 and 2002 , respectively. In 2005, he was appointed assistant professor in power systems at the University of Bologna where he was with the Power Systems laboratory until 2011. In 2010, he received the Associate Professor eligibility from the Politecnico di Milano, Italy. Currently, he is Associate Professor at the Swiss Federal Institute of Technology, Lausanne, Switzerland, where he accepted the EOS Holding Chair of the Distributed Electrical Systems laboratory. He is secretary and member of several IEEE and Cigré Working Groups. He was co-chairperson of the Technical Committee of the 9th edition of the International Conference of Power Systems Transients (IPST 2009) and of the 19th Power Systems Computation Conference (PSCC 2016). In 2013, he was the recipient of the IEEE EMC Society Technical Achievement Award. He is the Editor-in-Chief of the Elsevier journal Sustainable Energy, Grids and Networks and head of the Swiss Competence Center for Energy Research "FURIES". His research interests are in power systems with particular reference to real-time monitoring and operation of active distribution networks, integration of distributed energy storage systems, power system protections and power system transients. He is author and coauthor of over 220 scientific papers published in reviewed journals and international conferences. 